\title{
Coarse threat images reveal theta oscillations in the amygdala: A magnetoencephalography study
}

\author{
Frances A. Maratos \\ University of Derby, Derby, England \\ Karin Mogg and BRendan P. BRadley \\ University of Southampton, Southampton, England \\ AND \\ Gina Rippon and CARl Senior \\ Aston University, Birmingham, England
}

\begin{abstract}
Neurocognitive models propose a specialized neural system for processing threat-related information, in which the amygdala plays a key role in the analysis of threat cues. fMRI research indicates that the amygdala is sensitive to coarse visual threat relevant information - for example, low spatial frequency (LSF) fearful faces. However, fMRI cannot determine the temporal or spectral characteristics of neural responses. Consequently, we used magnetoencephalography to explore spatiotemporal patterns of activity in the amygdala and cortical regions with blurry (LSF) and normal angry, fearful, and neutral faces. Results demonstrated differences in amygdala activity between LSF threat-related and LSF neutral faces (50-250 msec after face onset). These differences were evident in the theta range $(4-8 \mathrm{~Hz})$ and were accompanied by power changes within visual and frontal regions. Our results support the view that the amygdala is involved in the early processing of coarse threat related information and that theta is important in integrating activity within emotion-processing networks.
\end{abstract}

It is now well established that the amygdala plays a key role in the processing of emotional information, regulates emotional responses, and controls fear reactions in a range of species (for reviews, see Aggleton \& Young, 2000; Davis \& Whalen, 2001; Phelps \& LeDoux, 2005; Zald, 2003). The amygdala is proposed to be part of a network of cortical and subcortical structures that allow efficient processing of, and rapid responding to, crude visual representations of potentially threatening stimuli (LeDoux, 1998, 2000). Within this network, cortical regions, including the ventral visual system, allow for the fine-grained analysis of stimuli and provide more refined, detailed information about the emotional significance of a perceived stimulus (Price \& Amaral, 1981; Winston, Vuilleumier, $\&$ Dolan, 2003). Frontal regions, such as the prefrontal cortex, are also proposed to play an important role in regulating amygdala activity in response to threat-related information (Bishop, 2007; Hariri, Mattay, Tessitore, Fera, \& Weinberger, 2003).

There is now considerable research that shows differential amygdala activation to threat-related cues compared with that to neutral cues (for a review, see Phan, Wager, Taylor, \& Liberzon, 2002). Such responses have also been found when awareness of the threat cue is restricted by masking (Williams, Das, et al., 2006) and when the threat- ening stimulus is unattended (Vuilleumier, 2005), but not always. That is, in contrast to Williams, Das, et al., Pessoa, Japee, Sturman, and Ungerleider (2006) showed that amygdala responses were greater for fearful than for neutral faces, but only when participants were able to reliably detect those stimuli. Amygdala activation has also been observed for both direct-threat cues (e.g., an angry face) and indirect-threat cues (e.g., a fearful face that signals threat in the environment, but is not threatening per se; Yang et al., 2002); there is, however, more consistent evidence for amygdala activation by fearful facial expressions (Davis \& Whalen, 2001; Phan et al., 2002; Whalen et al., 2001).

In recent neuroimaging work, the spatial frequency of stimuli has been manipulated in order to test the hypothesis that the amygdala response to threatening stimuli is driven by coarse visual information, which is primarily contained in low spatial frequencies (LeDoux, 2000). Using fMRI, Vuilleumier, Armony, Driver, and Dolan (2003) demonstrated increased activation of the amygdala in response to blurry, low spatial frequency (LSF) fearful faces compared with fine-grained, high spatial frequency (HSF) fearful faces. This finding contrasted with evidence of greater activation of the fusiform cortex by HSF faces compared with that by LSF faces, irrespective of their emotional ex- 
pressions. Such findings are consistent with the idea that the amygdala is particularly sensitive to crude visual representations of threat cues, such as those represented by LSF information (Vuilleumier \& Pourtois, 2007).

Neuroimaging research has shown that emotion processing depends on a network of neural structures, including the amygdala, as well as the frontal and visual cortices. Transient interactions between these structures underpin attentional and perceptual processing (Adolphs, 2002; Vuilluemier, 2005). However, fMRI does not have sufficient temporal resolution for investigating the time course of neural activity in these different regions. Adolphs suggested that the processing of emotional face stimuli occurs in the amygdala within $120 \mathrm{msec}$ of stimulus onset, which in turn modulates activity in the prefrontal and visual cortices. Pessoa, Kastner, and Ungerleider (2002) suggested, conversely, that emotion-modulation effects of the amygdala on cortical activity occur between 250 and $600 \mathrm{msec}$ after stimulus onset. That is, face stimuli initially elicit rapid face-selective processing in the visual cortex ( $170 \mathrm{msec})$; this does not depend on the emotional valence of those stimuli. Emotion-specific processing effects occur later, following feedback from the amygdala to the visual cortex (Pessoa et al., 2002, p. 43). This said, EEG research assessing event-related brain potentials (ERPs) suggests that emotion-specific effects of face stimuli occur in frontocentral cortical regions as early as 120 to $180 \mathrm{msec}$ poststimulus (Eimer \& Holmes, 2007). EEG methodologies have good temporal resolution, but they do not have sufficient spatial resolution to clarify which neural structures are responsible for such effects.

Another unresolved issue concerns the mechanisms responsible for integrating neural responses to emotional stimuli across subcortical and cortical structures, such as the amygdala and the frontal and visual cortices. In his recent dynamic-systems model, Lewis (2005) proposed that theta-band activity plays an important role in integrating and synchronizing activity within this neural network during emotion processing. Several studies have linked theta with emotional processing (for reviews, see Knyazev, 2007; Lewis, 2005; Paré, Collins, \& Pelletier, 2002). For example, Aftanas, Varlamov, Pavlov, Makhnev, and Reva (2001) demonstrated that theta-band activity is modulated by the affective valence of pictorial stimuli. Moreover, from a review of animal literature, Paré et al. concluded that amygdala neurons demonstrate theta-band activity during emotional arousal and that synchronized oscillatory activity in the amygdala may facilitate the consolidation of emotional memories. Reviewing such data, Lewis argued that "phase synchrony in the theta range may underpin the functional integration of systems mediating appraisal-emotion processes" (p. 189). Drawing upon evidence of strong connections between prefrontal and limbic structures (Miller, 1991), Lewis predicted that theta-band coupling between the amygdala and frontal cortices will occur when situations or tasks become emotionally relevant.

The main aim of this study was to use magnetoencephalography (MEG) to examine the temporal and spa- tial characteristics of neural responses to LSF threat and broad spatial frequency (BSF) threat-related information. Synthetic aperture magnetometry (SAM) was used to spatially map task-related power changes in brain activity because this technique is ideally suited for the analysis of oscillatory neuronal activity, which is not strictly time locked to stimulus onset (see the Method section). MEG has good temporal resolution, and previous research using MEG has reported activation effects of emotional stimuli (which included a mixture of fearful, happy, angry, surprised, and disgusted faces) in the amygdala and in the frontal and visual cortices within $200 \mathrm{msec}$ of stimulus onset (Streit et al., 2003). A recent MEG study published during the course of this investigation showed early synchronized activity $(<200 \mathrm{msec})$ in the gamma-band range in the amygdala and in the prefrontal and visual cortices in response to threat faces (Luo, Holroyd, Jones, Hendler, \& Blair, 2007). However, the Luo et al. study did not examine the role of theta in emotion processing. Nevertheless, such research has clearly demonstrated that MEG is sensitive to amygdala activity.

In the present study, the stimuli included angry, fearful, and neutral emotional facial expressions presented as both blurry (LSF) and normal (BSF) images. It was posited that the amygdala would show an early enhanced response to threat-related facial expressions, relative to neutral faces (i.e., within $250 \mathrm{msec}$ of face onset), and that this response would be accompanied by threat-related activation effects in prefrontal and visual areas. We predicted that these neural responses would be particularly evident for LSF threat faces because previous research has indicated that the amygdala is particularly sensitive to coarse affective information (Vuilleumier \& Pourtois, 2007). Our hypotheses were primarily concerned with neural activity in the theta range because Lewis's (2005) dynamic systems model predicts that phase locking of theta rhythms is important in the functional integration and temporal synchronization of subcortical and cortical systems involved in emotion processing.

\section{METHOD}

\section{Participants}

Nine participants ( 6 females, age range $=19-50$ years) with no history of neurological dysfunction or injury participated in the study. All participants had normal or corrected-to-normal vision (with contact lenses) and had an appropriate anatomical MR volume scan. Experimental procedures were in accordance with the Declaration of Helsinki and received approval from the University of Aston's ethical committee.

\section{Stimuli}

The stimuli (see Figure 1, top panel) included angry, fearful, and neutral faces selected from the NimStim Face stimulus set (macbrain .org/resource.htm). Eight actors (4 female) posing three different expressions (angry, fearful, neutral) were selected (models: 01, 03, $07,08,21,23,27,34)$. The stimuli were grayscaled, matched for size, and fitted to an oval shape $\left(2^{\circ} \times 3^{\circ}\right)$ with Adobe Photoshop 8.0 in order to obscure nonfacial features, such as hair and earrings. An in-house MATLAB script was then used to low-pass Gaussian filter the original BSF faces using a sigma parameter of 6 cycles/image. Prior to performing this filtering, the program standardized the BSF 

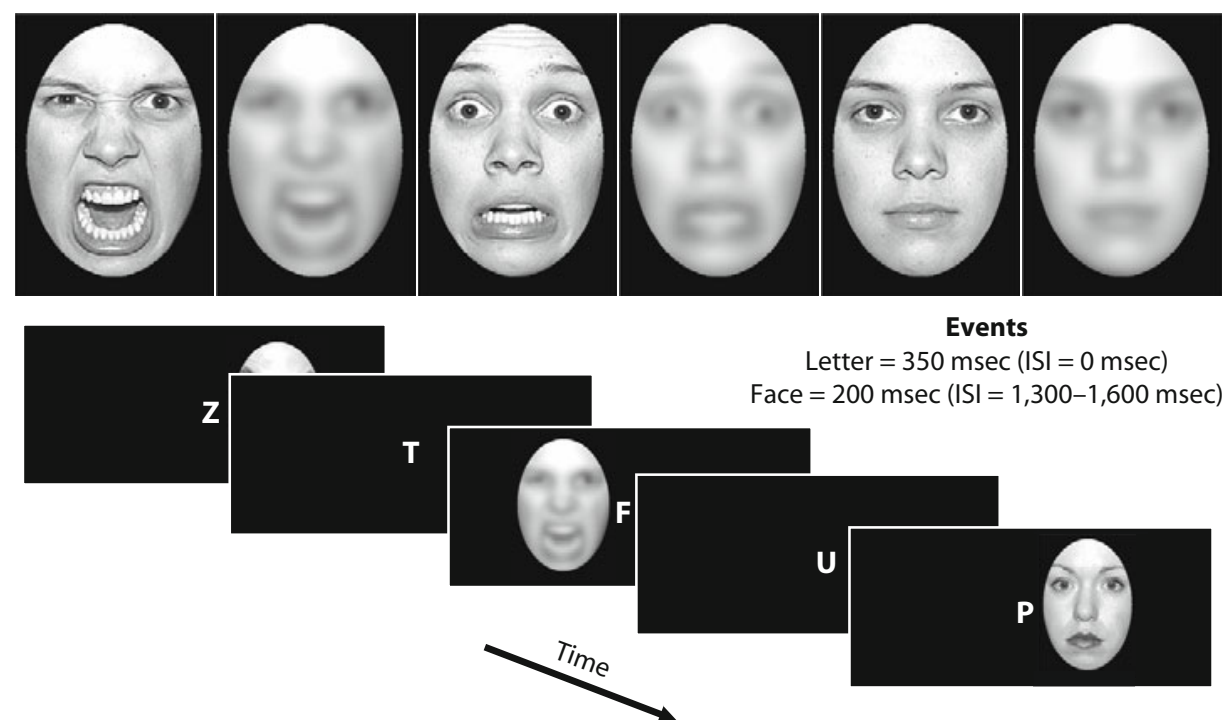

Figure 1. (Upper panel) Example of broad spatial frequency and low spatial frequency facial stimuli. (Lower panel) Example of stimulus events. Participants made a buttonpress response whenever the letter $\mathbf{X}$ appeared.

faces for luminance and contrast and cropped the stimuli so that each face was presented in an oval window on a black surround; there was an equivalent area across all images.

\section{Procedure}

Using a 275-channel, whole-head neuromagnetometer scanner, data were collected at a sampling rate of $625 \mathrm{~Hz}$, using a thirdorder gradiometer configuration with an antialiasing filter cutoff of $200 \mathrm{~Hz}$. The participants were seated in an upright position, and three electromagnetic coils were fastened to the participants' nasion and auricular points, respectively, to determine head position within the MEG helmet. The participants held a buttonpress response device, which they used with their dominant hand, in their lap. The display monitor was positioned outside the shielded room and was viewed through a window in the room, using a front-silvered mirror. The stimuli were viewed binocularly at an optical viewing distance of $2 \mathrm{~m}$. During the recording session, each participant's head was stabilized within the helmet, using an inflatable headcuff.

The sequence of trial events is shown in Figure 1 (lower panel). Throughout the task, a stream of letters was presented at central fixation (1 letter every $350 \mathrm{msec}$ ). The letters varied randomly, and the participants were asked to monitor the letter stream and indicate with a buttonpress response whenever the letter X appeared (10\% of trials). On each trial, a single face was presented for $200 \mathrm{msec}$ slightly off center, either to the left or right of the letter stream; stimulus eccentricity corresponded to a visual angle of $1.5^{\circ}$ (i.e., central vision). The interval between face offset on one trial and face onset on the next trial varied randomly between 1,300 and $1,600 \mathrm{msec}$. Data from trials displaying an $\mathrm{X}$ (or those in which a button response was recorded) were not analyzed. This task was used in preference to passive viewing in order to maintain an alert state of vigilance in the participants. It was also preferred over explicit emotional evaluation judgments of the faces because the latter might have attenuated amygdala activity (see, e.g., Critchley et al., 2000; Hariri et al., 2003).

For each stimulus type (e.g., LSF-angry), data from approximately 100 trials were recorded over two experimental runs that were $8 \mathrm{~min}$ in duration. Immediately after data acquisition, a Polhemus Isotrak 3-D digitizer was used to map the surface shape of each participant's head and localize the electromagnetic head coils with respect to that surface. These surface points were then coregistered with the individual participant's anatomical MRI using a surface matching procedure (Huppertz et al., 1998).

\section{Data Analysis}

Data from 2 participants were excluded from analysis due to excessive movement ( $>5 \mathrm{~mm}$ ). For the remaining 7 participants, data were bandpass filtered between 0.5 and $100 \mathrm{~Hz}$ and DC corrected according to a prestimulus baseline. In addition, a $50-\mathrm{Hz}$ powerline filter was applied, and all data were visually inspected, enabling the removal of trials with eyeblink (e.g., trials in which an extreme dipolar frontal pattern was observed to emerge within the recorded epoch) and/or movement artifacts (e.g., trials in which noise was observed for a period $\geq 25 \%$ of the recorded epoch). This resulted in the rejection of less than $10 \%$ of the data. SAM was then used to spatially map task-related power changes in oscillatory brain activity across participants. This method involved using fixed-array, weighted channels (as found in modern-day radar systems) to scan the brain for the sources of magnetic signals recorded at the scalp (for a review, see Hillebrand, Singh, Holliday, Furlong, \& Barnes, 2005). In consequence, SAM requires no a priori assumptions about the number of sources activated and is ideally suited for the analysis of neuronal activity that is not strictly time locked to stimulus onset (i.e., induced activity).

It has been questioned whether MEG allows for the estimation of sources in deep subcortical structures, such as the amygdala (since MEG is often portrayed as a method with excellent temporal resolution but limited spatial resolution). However, there have now been several demonstrations of signal detection from deep structures, such as the amygdala and the hippocampus, using evoked field measures with MEG (e.g., Ioannides, Poghosyan, Dammers, \& Streit, 2004; Rogers et al., 1991; Streit et al., 2003; Tesche, 1997). Both Tesche and Moradi et al. (2003) provided stringent validations of these methodologies. More recently, oscillatory activity has been recorded from both the hippocampus (e.g., Tesche \& Karhu, 2000) and the amygdala (e.g., Luo et al., 2007), using MEG. As with our research, in the latter study the adaptive beamformer SAM was used. Given that this methodology is both spatially sensitive to correlated activity and less sensitive to noise artifacts - problems associated with localizing activity from deep sources - SAM may indeed be a better detector of deep sources than the nonadaptive 


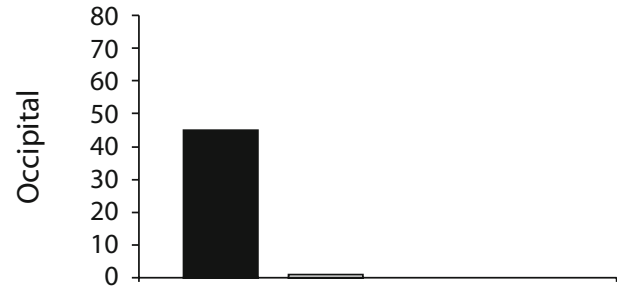

$(50-250 \mathrm{msec})$

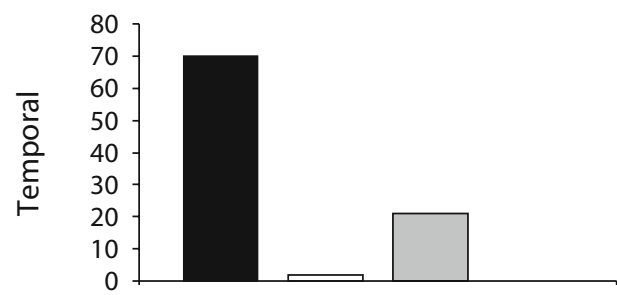

(50-250 msec)

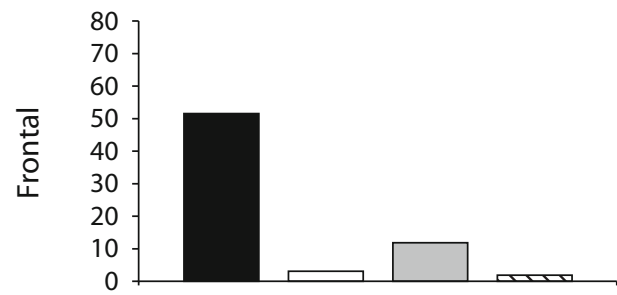

$(50-250 \mathrm{msec})$

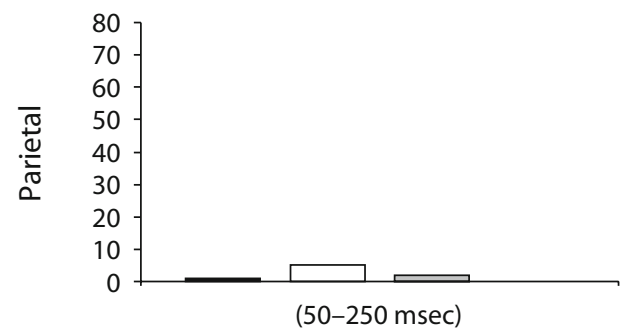

Theta $\quad$ Alpha

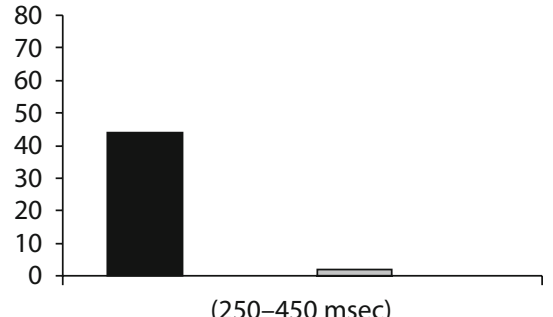

$(250-450 \mathrm{msec})$
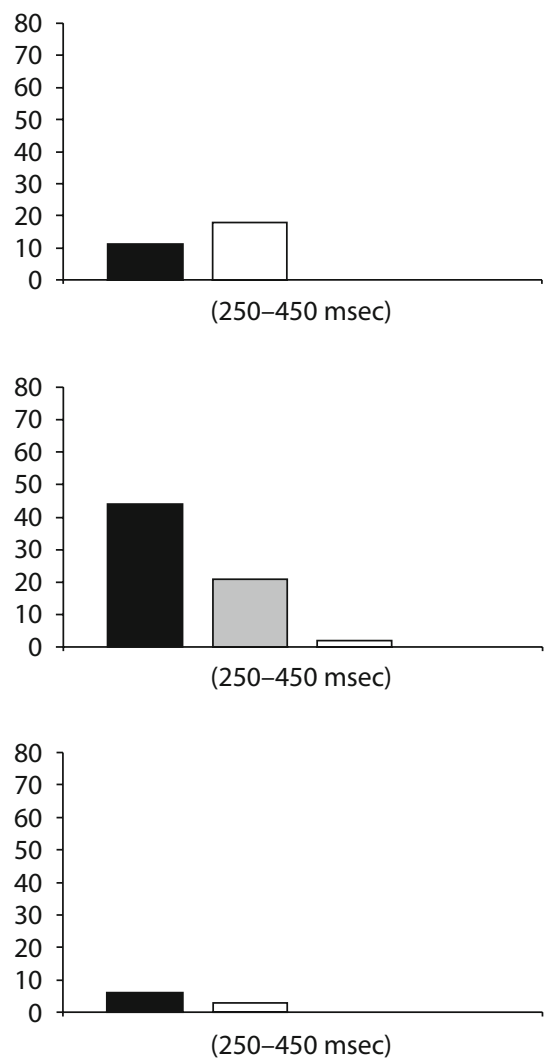

Beta

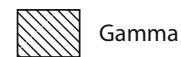

Figure 2. The percentage of active voxels for each frequency band across the early $(50-250 \mathrm{msec})$ and late $(250-450 \mathrm{msec})$ time windows for the low spatial frequency threat versus neutral comparisons. The early time window shows a greater number of active voxels in the theta band across the occipital temporal frontal regions. A time window (50-250 msec) $\times$ frequency (theta, 4-8 Hz; alpha, 8-13 Hz; beta, 10-20 Hz; low gamma, 20-30 Hz) ANOVA with number of active voxels as the dependent variable revealed a main effect of frequency band. The significant effect of frequency band on the number of active voxels was evident within both the early and late time windows.

(but validated) techniques discussed above (see Luo et al., 2007; Vrba \& Robinson, 2001).

In brief, when using SAM, for each voxel in a predefined source space, an optimal spatial filter that linked activity in that voxel to the MEG system's sensor array was constructed. In the present study, each participant's MRI was divided into voxels of $5 \times 5 \times 5 \mathrm{~mm}$ (5-mm grids). The filter output for each voxel was calculated independently, as the weighted sum of the sensor signals, yielding a measure of current density as a function of time (for a more detailed description of the spatial filter construction, see Maratos, Anderson, Hillebrand, Singh, \& Barnes, 2007). This procedure is conceptually similar to using an electrode at the neural source location, and, for this reason, the spatial filter is often called a virtual electrode (Barnes \& Hillebrand, 2003).
A boxcar experimental design was used, in which spectral power changes between matching 50 - to $250-\mathrm{msec}$ and 250 - to 450 -msec poststimulus onset time windows were calculated for several traditional frequency bands (theta, 4-8 Hz; alpha, 8-13 Hz; beta 1, 10-20 Hz; beta 2, 20-30 Hz; gamma 1, 30-40 Hz; gamma 2, 40$100 \mathrm{~Hz}$ ). These 200-msec time windows were used for all comparisons (i.e., threat-related vs. neutral expressions, as well as angry vs. fearful expressions, for both BSF and LSF stimuli) because they (1) produced sufficient temporal data for investigating power differences at the lowest and highest frequencies and (2) were comparable to previous research noted earlier (i.e., that $250 \mathrm{msec}$ represents an informative distinction in emotion processing; see Adolphs, 2002; Eimer \& Holmes, 2007; Pessoa, 2005; Williams, Palmer, Liddell, Song, \& Gordon, 2006). Note, however, that since altering the spa- 
tial frequency properties of visual stimuli has been shown to moderate gamma-band activity (Hadjipapas, Adjamian, Swettenham, Holliday, \& Barnes, 2007), analyses of the 40- to 100-Hz gamma response to LSF stimuli were not undertaken, since it would have been difficult to interpret the results.

For each participant, time window, and frequency band, the difference between spectral power estimates was assessed for every voxel, using a pseudo- $t$ statistic (Robinson \& Vrba, 1999) enabling a 3-D SAM image of activity to be generated for each individual. Data were then normalized, and nonparametric permutation analyses (Nichols \& Holmes, 2002; Singh, Barnes, \& Hillebrand, 2003) were performed with the SnPM toolbox (www.sph.umich .edu/ni-stat/snpm) to assess significant group effects for voxel-level inferences. Nonparametric permutation analyses (i.e., SnPM) were adopted, given that the assumption of normality was not required. Thus, such analyses, unlike random- and fixed-effects models, are suited for the robust analysis of data with low degrees of freedom (see Singh et al., 2003). A synthetic third-order gradiometer design was also employed in order to increase the signal-to-noise ratio. Additionally, since the SnPM procedure we employed included the use of a probability distribution map generated by the largest $t$ values in the volume (rather than the $t$ values at each voxel), the problem of multiple comparisons was circumvented (for more information on SnPM analyses, see Nichols \& Holmes, 2002).

Probability maps for significant group effects $(p<.05$, corrected) were visualized using mri3 dX (imaging.aston.ac.uk/ $\mathrm{mri3} \mathrm{dX} /$ ) and regions of interest (ROIs) determined from the SnPM significant voxel clusters. Once significant ROIs were established using a data-driven approach, a theory-driven approach was adopted to restrict supplementary exploratory analyses to regions previously implicated in emotion processing. To examine the power and timecourse differences of any changes in oscillatory (i.e., induced) activity within these ROIs, time-frequency plots were calculated for all individuals, using a Morlet wavelet transform. These plots were created from single-trial activation waveforms for a given ROI, and, from these, average and group average wavelet time-frequency/ power-change plots were created. These revealed percentage change in energy per time-frequency bin relative to the prestimulus phase. To establish that activity present in these plots was induced and not evoked, additional time-frequency plots created from the average of the activation waveforms for each ROI were produced to demar- cate any evoked activity. These plots revealed amplitude change per time-frequency bin relative to baseline.

\section{RESULTS}

\section{Group Data}

For the 50- to $250-\mathrm{msec}$ and $250-$ to $450-\mathrm{msec}$ time periods, SnPM voxel-level analyses $(p<.05$, corrected) were computed for each emotion contrast (i.e., angry vs. neutral, fearful vs. neutral, angry vs. fearful) within each frequency band (i.e., 4-8 Hz, 8-13 Hz, 10-20 Hz, $20-30 \mathrm{~Hz}, 30-40 \mathrm{~Hz}, 40-100 \mathrm{~Hz}$ ). These contrasts were examined separately for LSF and BSF faces (with the exception of the 40- to $100-\mathrm{Hz}$ band for LSF faces, as noted earlier). For the BSF comparisons (e.g., BSF-angry vs. BSF-neutral, BSF-fearful vs. BSF-neutral), there were no significant results in the theta range, which was of primary interest here. Some activation differences were observed for the BSF contrasts, but these were limited, did not involve the amygdala, and were restricted to the visual cortices and gamma frequency range $(40-100 \mathrm{~Hz})$; these data are reported elsewhere.

For the LSF threat comparisons (i.e., LSF-angry vs. LSF-neutral, LSF-fearful vs. LSF-neutral), the SnPM analyses revealed significant differences in brain activity in several frequency bands (e.g., $4-8 \mathrm{~Hz}, 8-13 \mathrm{~Hz}$, $10-20 \mathrm{~Hz}, 20-30 \mathrm{~Hz}$ ) and a range of brain regions (e.g., limbic system, visual cortices, prefrontal cortex). These data are illustrated in Figure 2, in which the percentage of active voxels is presented as a function of frequency band, time window, and brain lobe. Consistent with the predictions of Lewis (2005), the early time window shows a greater number of active voxels in the theta band across the occipital temporal frontal regions, and a time window $(50-250 \mathrm{msec}, 250-450 \mathrm{msec}) \times$ frequency band $(4-8 \mathrm{~Hz}, 8-13 \mathrm{~Hz}, 10-20 \mathrm{~Hz}, 20-30 \mathrm{~Hz})$

Table 1

SnPM Group SAM Theta Results $(p<.05$, Corrected) for the LSF-Threat (Angry, Fearful) Versus LSF-Neutral 50- to 250-msec Time-Window Comparisons

\begin{tabular}{|c|c|c|c|c|c|}
\hline Brain Region & $x$ & $y$ & $z$ & $p$ & No. of Voxels \\
\hline \multicolumn{6}{|l|}{ Angry vs. Neutral Stimuli } \\
\hline L. middle frontal gyrus (BA 10), frontal lobe & -36 & 57 & 0 & .008 & 1,670 \\
\hline Including L. superior frontal gyrus & -20 & 57 & -9 & & \\
\hline R. superior temporal gyrus (BA 21), temporal lobe & 60 & -24 & -3 & .008 & 1,504 \\
\hline Including R. amygdala & 21 & -9 & 12 & & \\
\hline C. cuneus (BA 18), occipital lobe & 0 & -105 & 15 & .008 & 117 \\
\hline R. cuneus (BA 19), occipital lobe & 15 & -96 & 36 & .016 & 106 \\
\hline R. medial frontal gyrus (BA 10), frontal lobe & 12 & 63 & 0 & .016 & 234 \\
\hline R. declive, cerebellum & 39 & -84 & -27 & .016 & 483 \\
\hline L. lingual gyrus, sublobar & -24 & -60 & -3 & .016 & 666 \\
\hline Including $\mathrm{L} . \mathrm{V} 1$ & -13 & -63 & -10 & & \\
\hline L. subgyral, frontal lobe & -15 & -30 & 60 & .016 & 114 \\
\hline R. middle occipital gyrus, occipital lobe & 54 & -69 & -9 & .016 & 71 \\
\hline R. middle frontal gyrus, frontal lobe & 63 & 6 & 51 & .016 & 26 \\
\hline R. inferior frontal gyrus, frontal lobe & 42 & 30 & -18 & .023 & 102 \\
\hline \multicolumn{6}{|l|}{ Fearful vs. Neutral Stimuli } \\
\hline L. frontal lobe (unidentified) & -27 & 54 & 48 & .016 & 15 \\
\hline R. subgyral, frontal lobe & 24 & 9 & 27 & .039 & 39 \\
\hline L. precuneus, parietal lobe & -27 & -63 & 42 & .039 & 18 \\
\hline
\end{tabular}

Note-The location of the most significant voxel within each identified area is reported. 
ANOVA with number of active voxels as the dependent variable revealed a significant main effect of frequency band $[F(3,18)=11.02, p<.001]$. For each time window, a one-way ANOVA of the number of active voxels with frequency band $(4-8 \mathrm{~Hz}, 8-13 \mathrm{~Hz}, 10-20 \mathrm{~Hz}, 20-30 \mathrm{~Hz})$ as the independent variable further revealed that the main effect of frequency was driven by a greater number of active voxels in the late time window $[F(3,12)=4.32$, $p=.03]$ and by a highly significant difference in the early time window $[F(3,12)=6.23, p=.009]$. Moreover, within the amygdala, SnPM-significant differences in power were observed only in the theta bandwidth. Accordingly, the results focus on analyses of LSF face data in the theta frequency range.

\section{LSF-Threat Versus LSF-Neutral Activation Differences at 4-8 Hz}

The 50- to 250-msec time window. The group results revealed a range of power changes within cortical and subcortical regions for both the LSF-angry versus LSF-neutral comparison and the LSF-fearful versus LSFneutral comparison. The neuroanatomical locations of significant peak SnPM voxels are reported in Table 1. Of importance, clusters of significant voxels were observed in the amygdala (and temporal lobes), primary visual areas (including V1), and prefrontal regions (including the superior frontal gyrus, or SFG, within the orbitofrontal cortex) for at least one of the threat versus neutral comparisons, if not both. These power changes always reflected a reduction in power for the LSF-threat expressions compared with the LSF-neutral expressions and are displayed in the upper panel of Figure 3 for the LSF-angry versus LSF-neutral comparison.

The 250- to 450-msec time window. The group results revealed a range of power changes within cortical and subcortical regions for both the LSF-angry versus LSF-neutral and LSF-fearful versus LSF-neutral comparisons (see Table 2 for the neuroanatomical locations of significant peak SnPM voxels). Again, changes always reflected a reduction in power for the LSF-threat expressions compared with LSF-neutral expressions. However, whereas clusters of significant voxels were still observed in prefrontal regions (including the inferior frontal gyrus, or IFG, within the orbitofrontal cortex) for both threat versus neutral comparisons, differences in activity were no longer evident in either the amygdala or the primary visual area. For results from the LSF-angry versus LSF-neutral comparison, see the lower panel of Figure 3.

\section{LSF-Angry Versus LSF-Fearful Activation Differences at 4-8 Hz}

No significant differences in power were observed for the contrast of LSF-angry versus LSF-fearful faces for either the 50- to 250 -msec or 250- to 450-msec time window. However, the results shown in Tables 2 and 3 suggest that compared with LSF-neutral faces, LSF-angry faces resulted in more significant voxel clusters (especially in the early time window) than did LSF-fearful faces.
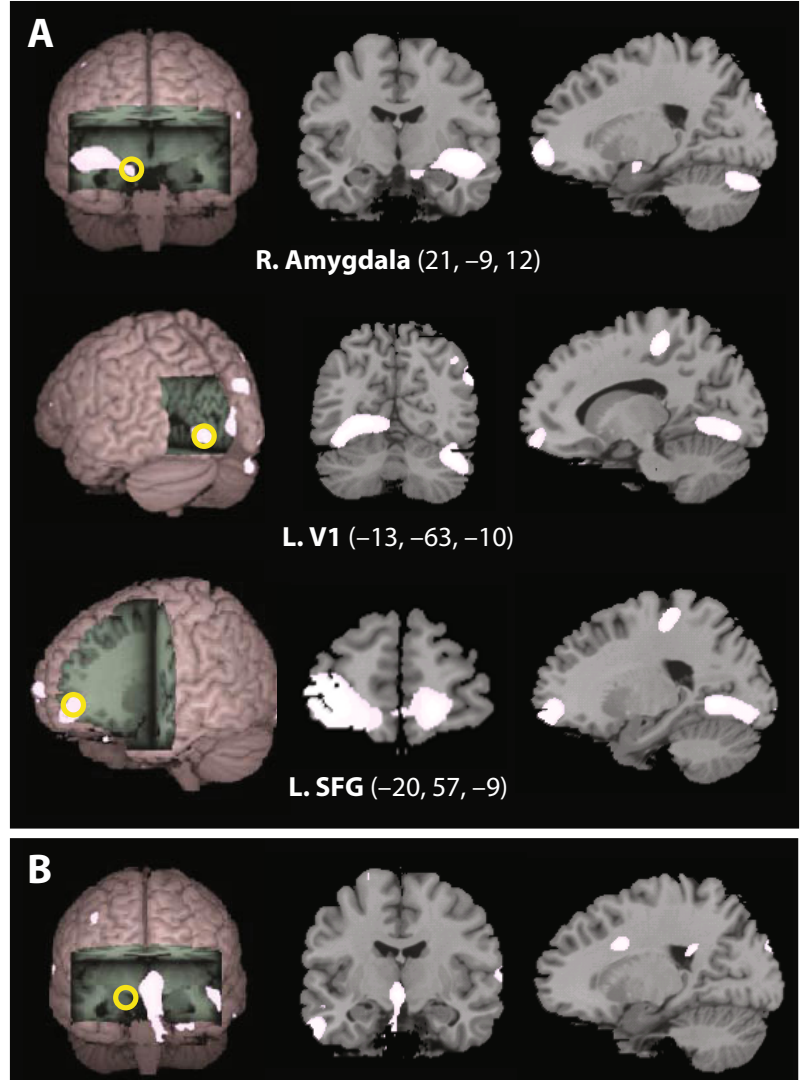

R. Amygdala $(21,-9,12)$

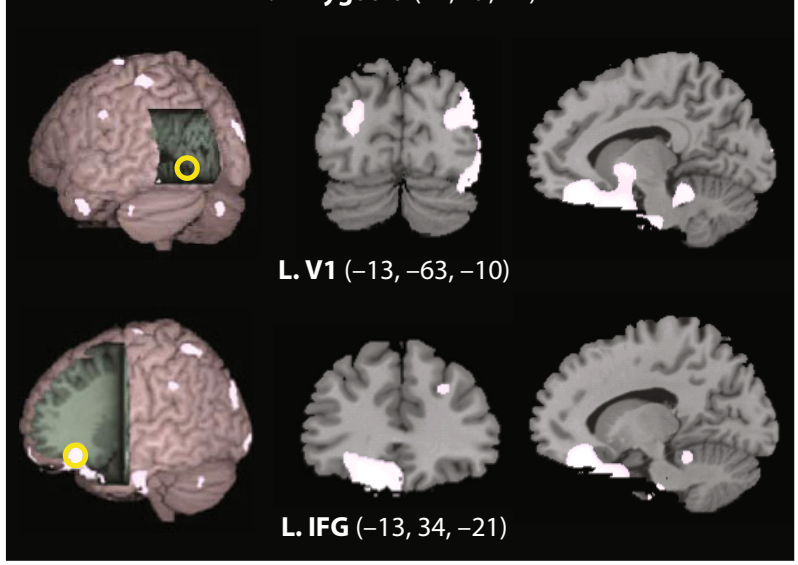

95\% Confidence Interval

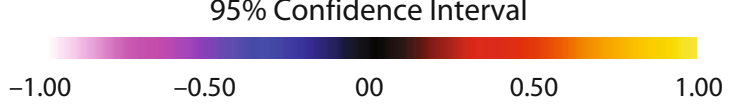

Figure 3. (A) Surface-rendered, axial, and sagittal SnPM group SAM images for the LSF-angry versus LSF-neutral, 50- to 250msec theta time window comparison (data thresholded at the $p<$ .05 level). In the amygdala, primary visual areas, and prefrontal cortices, significant reductions in source power were observed for angry faces relative to those for neutral faces. (B) Surfacerendered, axial, and sagittal SnPM group SAM images for the LSF-angry versus LSF-neutral, 250- to 450 -msec theta time window comparison. In the prefrontal cortices, significant reductions in source power are still observed for angry faces relative to those for neutral faces. However, in the amygdala and primary visual areas, there are now no differences in power between angry faces and neutral faces. 
Table 2

SnPM Group SAM Theta Results $(p<.05$, Corrected) for the LSF-Threat (Angry, Fearful) Versus LSF-Neutral 250- to 450-msec Time Window Comparisons

\begin{tabular}{|c|c|c|c|c|c|}
\hline Brain Region & $x$ & $y$ & $z$ & $p$ & No. of Voxels \\
\hline \multicolumn{6}{|l|}{ Angry vs. Neutral Stimuli } \\
\hline R. superior frontal gyrus (BA 11), frontal lobe & 6 & 60 & -21 & .008 & 1,662 \\
\hline Including L. inferior frontal gyrus & -13 & 34 & 21 & & \\
\hline R. superior temporal gyrus (BA 22), temporal lobe & 78 & -9 & 6 & .016 & 132 \\
\hline R. middle occipital gyrus (BA 19), occipital lobe & 54 & -84 & 6 & .016 & 632 \\
\hline L. subgyral, temporal lobe & -48 & -3 & -15 & .016 & 259 \\
\hline L. middle occipital gyrus, occipital lobe & -21 & -105 & -3 & .016 & 50 \\
\hline L. cuneus (BA 19), occipital lobe & -24 & -105 & -3 & .016 & 253 \\
\hline L. inferior parietal lobule (BA 40), parietal lobe & -39 & -57 & 63 & .016 & 121 \\
\hline Ulva, cerebellum & 6 & 105 & -24 & .016 & 85 \\
\hline Precentral gyrus (BA 6), frontal lobe & -24 & -18 & 78 & .016 & 76 \\
\hline \multicolumn{6}{|l|}{ Fearful vs. Neutral Stimuli } \\
\hline L. pons, brain stem & -12 & -21 & -39 & .016 & 283 \\
\hline R. superior frontal gyrus (BA 6), precentral gyrus & 21 & -15 & 84 & .016 & 62 \\
\hline L. superior frontal gyrus (BA 10), frontal lobe & -9 & 66 & 33 & .023 & 51 \\
\hline L. superior parietal lobe (BA 7), parietal lobe & -33 & -54 & 69 & .023 & 58 \\
\hline C. superior parietal lobe (BA 7), parietal lobe & -6 & -75 & 63 & .023 & 46 \\
\hline L. superior temporal gyrus (BA 8), temporal gyrus & -42 & 9 & -12 & .031 & 27 \\
\hline L. middle temporal gyrus (BA 21), temporal lobe & -63 & -3 & -27 & .039 & 29 \\
\hline L. parahippocampal gyrus, limbic lobe & -33 & -18 & -42 & .047 & 17 \\
\hline L. inferior frontal gyrus (BA 9), frontal lobe & -51 & 6 & 30 & .047 & 10 \\
\hline R. precuneus, parietal lobe & 36 & -75 & 36 & .047 & 10 \\
\hline
\end{tabular}

Note-The location of the most significant voxel within each identified area is reported.

\section{Supplementary Single-Subject-Based Data Analyses}

The main reasons for investigating data at the singlesubject level were (1) to examine in detail the nature of the significant power differences between the threat and neutral expressions within each key region, such as the right amygdala or the SFG, identified in the group SnPM analyses and (2) to explore the time course of activity within those regions where SnPM-significant differences were observed (e.g., the 50- to 250-msec time window). In these analyses, therefore, prestimulus activity was directly compared with poststimulus activity for each type of LSF facial expression.

For all participants, time-frequency analyses were conducted for bilateral voxels in the amygdala, visual cortices (primary visual cortex, V1), and frontal cortices, such as the SFG and the IFG. From these data, percent power changes were calculated in order to provide an overview of power increases and/or decreases after stimulus onset, relative to the prestimulus phase for the theta band. Whereas the ROIs were primarily informed by the SnPM analyses (see Tables 1 and 2), activations observed in previous research were used to restrict analyses to brain regions that have been implicated in emotion processing (Adolphs, 2002; Phillips, Drevets, Rauch, \& Lane, 2003; Vuilleumier \& Pourtois, 2007). Given the presumed importance of the fusiform gyrus (FFG) in the processing of facial structure, an ROI analysis of activity in this area was also undertaken. To avoid confounds associated with using normalized MRI brain templates (Woods, 1996), in all of the above supplementary analyses, voxels within the ROIs were generated using data from each participant's original MRI SAM image.

Plots depicting induced activity within the amygdala, V1, SFG, and FFG are shown in Figures 4 and 5 (time- frequency and power plots, respectively). These plots depict mean power changes at a given ROI (i.e., virtual electrode) for every individual and are averaged across the group. In each plot, the stimulus appeared on-screen at Time 0. In Figure 4, red and blue represent percent change in power. In all cases, significant differences are indicated with the dashed square.

Amygdala activity. The time-frequency plots reveal that for the angry facial expressions (Ang), a reduction in power $(5-7 \mathrm{~Hz})$ is observed following stimulus onset in the right amygdala. Of importance, a similar pattern of decreased power is not present in the time-frequency plots for the neutral facial expressions. Indeed, for the 50to 250-msec time period where significant SnPM differences were observed (see Table 1), the power plot reveals that power tended to decrease for angry expressions (red line) and increase for neutral expressions (blue line), and that this differential response appeared within $100 \mathrm{msec}$ of stimulus onset.

V1 activity. For angry expressions, the time-frequency plots reveal a brief bilateral reduction in power $(\sim 4-6 \mathrm{~Hz})$ soon after stimulus onset. For the 50- to 250 -msec time period where these differences were found to be significant, the power plot shows a short-lasting decrease in power (peaking at $\sim 100 \mathrm{msec}$ ) for the angry expressions, which was absent for the neutral expressions.

Frontal cortex activity. For angry expressions, a bilateral reduction in power $(5-7 \mathrm{~Hz})$ is observed in the timefrequency plots following face onset in superior frontal regions. However, for the neutral expressions, the early power changes are less marked. Indeed, for the 50- to 250msec time window, where results were found to be significant, both plot types demonstrated a power decrease for angry expressions but a power increase for neutral expres- 


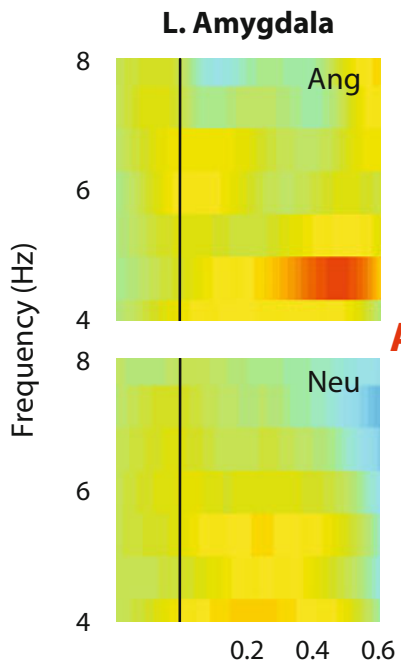

A

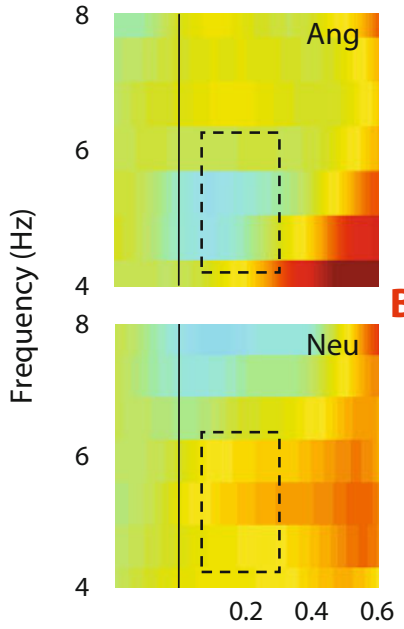

R. Amygdala
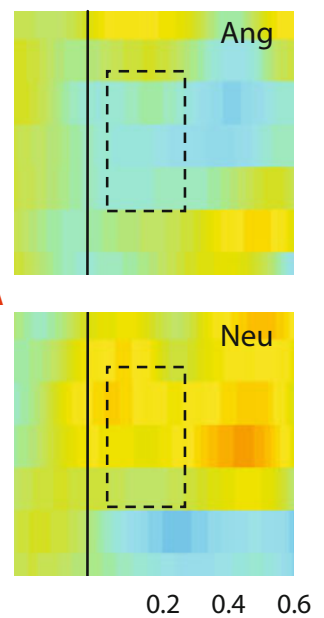

R. V1

L. SFG
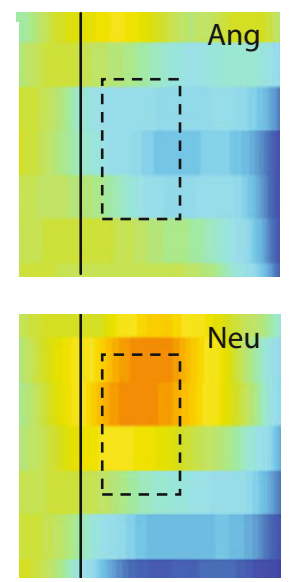

$0.2 \quad 0.4 \quad 0.6$

L. FFG

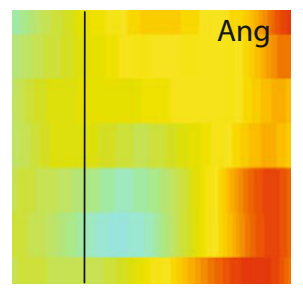

B

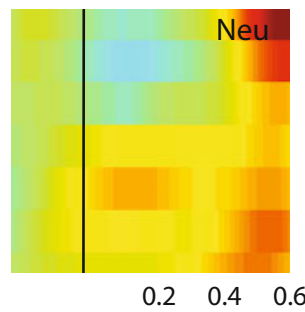

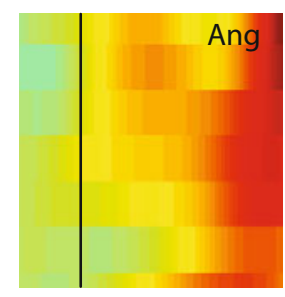

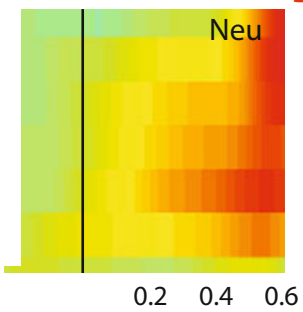

R. SFG

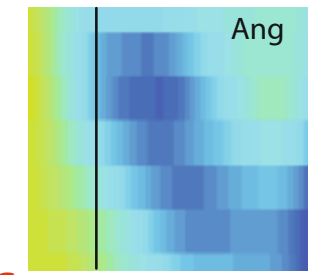

C

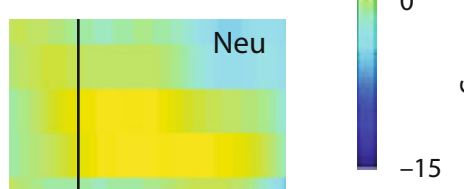

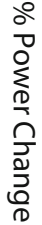

Figure 4. Group time-frequency wavelet plots for LSF faces computed for an ROI within the (A) amygdala, (B) V1, (C) SFG, and (D) FFG (Ang, angry faces; Neu, neutral faces). In all cases, the face stimulus appeared on-screen at Time 0 (black line), and the plots show percent change in energy per time-frequency bin relative to the prestimulus phase. Additionally, for panels $\mathrm{A}, \mathrm{B}$, and $\mathrm{C}$, the dashed boxes within the wavelet plots highlight the time window and brain region where significant SnPM differences were found.

A

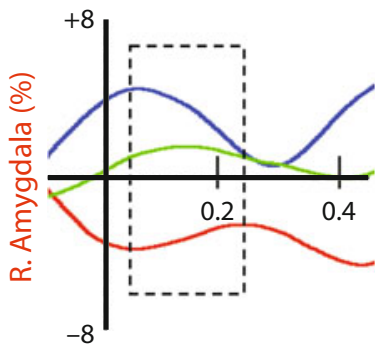

B

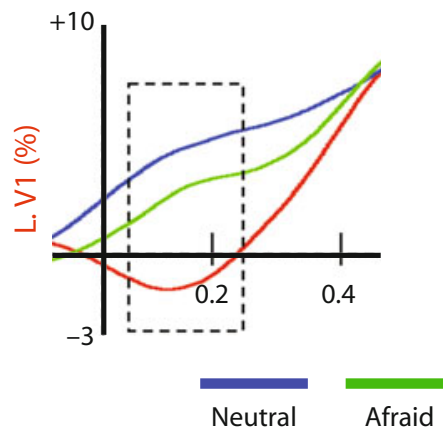

C

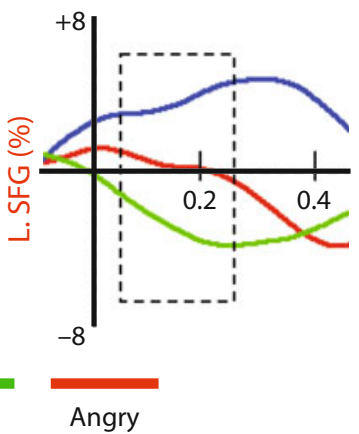

D

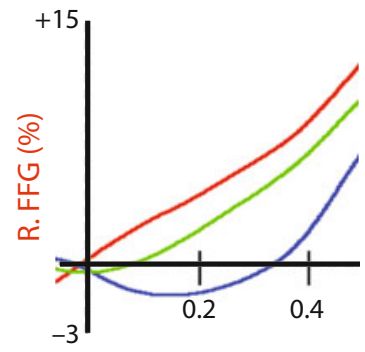

Figure 5. Average power change plots for an ROI in (A) right amygdala, (B) left V1, (C) left SFG, and (D) right FFG. Activity for neutral faces is indicated with the blue line, for fear faces with the green line, and for angry faces with the red line. In all cases, the face stimulus appeared on the screen at Time 0 (dashed line). Significant differences between conditions (e.g., angry vs. neutral) are indicated with the dashed square. Although no significant differences were revealed within the FFG, a plot is included for reference. 
sions. The pattern of activity within the IFG (results not shown) was similar - albeit slightly delayed in time - to those observed within the SFG, consistent with the results reported in Table 2.

FFG activity. Although nonsignificant, timefrequency and power plots for induced activity within the FFG are shown in Figures 4 and 5 for reference.

\section{DISCUSSION}

The main aim of the present study was twofold. First, we set out to investigate the spatiotemporal characteristics of neural responses to threat-related informationnamely, LSF (blurry) and BSF depictions of angry and fearful facial expressions. Second, we wished to examine whether oscillations within the theta range could underlie activity in emotion-processing networks, because it has been predicted that synchrony in the theta range may underpin processes of emotion (Lewis, 2005). Related to these aims were three main findings. First, results indicated that within $250 \mathrm{msec}$ of stimulus onset, there were power decreases in the amygdala and the visual and frontal cortices for LSF-threat faces relative to those for LSF-neutral faces (but not for BSF-threat faces relative to those for BSF-neutral faces). Second, all findings were observed in the theta power range. And third, within the amygdala, these power decreases were lateralized to the right nuclei.

Previous research has indicated that the amygdala plays a critical role in emotion processing as part of a neural network that also includes frontal and visual processing regions, and that it may be particularly responsive to crude representations of threat (Vuilleumier \& Pourtois, 2007; Winston et al., 2003). fMRI does not have sufficient temporal resolution to investigate the time course of amygdala responses to threat cues; utilizing MEG, however, we were able to observe emotional processing of threat-related information within the theta band within $250 \mathrm{msec}$ of stimulus onset in a network of areas involving the amygdala and the visual and frontal cortices. The spatiotemporal pattern of activity at the virtual electrode level further suggested that differential activity in the amygdala and visual cortices occurred soon after stimulus onset (i.e., within $100 \mathrm{msec}$ ). Thus, the present results are compatible with the temporal model of emotional-face recognition proposed by Adolphs (2002), in which it is argued that within the amygdala, the processing of highly salient emotional stimuli occurs within $120 \mathrm{msec}$, which subsequently provides early modulation of perceptual and attentional processing (see also Kawasaki et al., 2001; cf. Pessoa, 2005).

Recent fMRI work has further indicated that perceptual processing in a network of areas involving the amygdala is primarily driven by the low spatial frequency of stimuli (Vuilleumier et al., 2003). The findings of the present study add to this existing literature insofar as analyses of group data revealed that differences in neuronal activity within the amygdala and frontal regions for threat-related expressions compared with neutral expressions were only observed for the LSF faces. Additionally, our data are consistent with those from research by Öhman, Carlsson, Lundqvist, and Ingvar (2007), in which it is proposed that activation of the amygdala facilitates low-level visual processing, such as that associated with unattended and/ or degraded (e.g., blurry) threat-related stimuli, as in the present research.

The present study also adds to a growing body of ERP literature in which it is suggested that early responses to emotionally valenced face stimuli may reflect processing in a thalamocortical neural system parallel to that associated with structural-face encoding in ventral-visual areas and the robust N170 (for a review, see Eimer \& Holmes, 2007; Williams, Palmer, et al., 2006).

SnPM analyses of power changes in the two time windows revealed that significant differences in amygdala activity for the threat versus neutral comparisons were not sustained in the later time window (i.e., $250-450 \mathrm{msec}$ ). Still, a significant reduction in power was observed for the threat stimuli during this time period in some frontal regions, such as the IFG (as shown in Table 2 and in the bottom panel of Figure 3). Previous research has suggested that amygdala-frontal interactions play a modulatory feedback role, with frontal regions attenuating amygdala responses to threat (e.g., Hariri et al., 2003; Phillips et al., 2001). Thus, the frontal activity observed for the threat compared with neutral stimuli in the later time window could reflect effortful emotional processes related to the appraisal of threatening stimuli, including emotional regulation and/or response suppression (see also Ochsner \& Gross, 2005).

Lewis (2005) has proposed that theta activity may play a primary role in integrating and synchronizing activity within and between such brain regions implicated in emotion processing (e.g., the amygdala and the visual and frontal regions). We observed all changes to be greatest or only present in the theta range, so our findings are compatible with this view. In consequence, the present findings also have important implications concerning the neuronal mechanisms that are responsible for coordinating processes of emotion across brain regions - especially since relatively little is known about such mechanisms.

Thus, the main findings of the present research are compatible with recent theoretical views of emotion processing (Adolphs, 2002; Lewis, 2005; Phillips et al., 2003), but a number of additional, unpredicted findings warrant consideration. For example, why was amygdala activity observed for the LSF faces only? Given that BSF faces include LSF information (as well as HSF information), why did they not elicit a similar pattern of neuronal activation? It has been previously suggested that the amygdala shows greater activation to potential-threat stimuli when they are ambiguous (Adams, Gordon, Baird, Ambady, \& Kleck, 2003; Davis \& Whalen, 2001; Whalen et al., 2004). This could explain why in the present study, amygdala activation was easier to detect for ambiguous emotional stimuli (blurry LSF-threat faces) than for unambiguous stimuli (BSF-threat faces) - especially with small numbers of participants. With regard to this, a caveat is that data were analyzed from a small number of participants only (i.e., <10). Several recent fMRI and 
MEG imaging studies have been published with low numbers of participants (e.g., Hadjipapas et al., 2007; Liu \& Ioannides, 2006; Suslow et al., 2006); but, where feasible, larger participant populations should be sought. This would also allow investigation of individual differences in emotion processing, such as influences of anxiety, gender, or genotype (Bishop, 2007; Hamann \& Canli, 2004).

For the LSF faces, we further observed that threatrelated effects within the amygdala tended to be restricted to the right hemisphere. Results from previous hemodynamic imaging studies have similarly revealed evidence of amygdala lateralization during emotion processing (for a review, see Baas, Aleman, \& Kahn, 2004), and it has been hypothesized that right amygdala activity is associated with fast, shallow analysis of affect-related information (Gläscher \& Adolphs, 2003; Markowitsch, 1998; Wright et al., 2001). Our data are compatible with the view that the right amygdala plays an important role in processing coarse threat related information.

In sum, the present MEG results extend previous fMRI research and support the hypothesis that coarse threat related visual inputs lead to early activation (i.e., within $250 \mathrm{msec}$ of stimulus onset) of the amygdala and of a network of cortical areas involved in emotion processing. Also of significance, our study indicates that activity in the theta range may underpin the functional integration of emotion-processing systems within an amygdala, visual, and prefrontal network.

\section{AUTHOR NOTE}

This research was funded in part by Wellcome Trust Grant 051076 to K.M. and B.P.B. We thank Mark Georgeson, Luke Phillips, Sian Worthen, and Arjan Hillebrand for assistance, as well as the anonymous reviewers who provided suggestions on earlier drafts of this article. Correspondence concerning this article should be addressed to F. A. Maratos, Centre for Psychological Research, Department of Psychology, University of Derby, Derby DE22 1GB, England (e-mail: f.maratos@ derby.ac.uk).

\section{REFERENCES}

Adams, R. B., Jr., Gordon, H. L., Baird, A. A., Ambady, N., \& Kleck, R. E. (2003). Effects of gaze on amygdala sensitivity to anger and fear faces. Science, 300, 1536.

Adolphs, R. (2002). Neural systems for recognizing emotion. Current Opinion in Neurobiology, 12, 169-177.

Aftanas, L. I., Varlamov, A. A., Pavlov, S. V., Makhnev, V. P., \& REvA, N. V. (2001). Affective picture processing: Event-related synchronization within individually defined human theta band is modulated by valence dimension. Neuroscience Letters, 303, 115-118.

Aggleton, J. P., \& Young, A. W. (2000). The enigma of the amygdala: On its contribution to human emotion. In R. D. Lane \& L. Nadel (Eds.), Cognitive neuroscience of emotion (pp. 106-128). Oxford: Oxford University Press.

BAAs, D., AlEMAN, A., \& KAHN, R. S. (2004). Lateralization of amygdala activation: A systematic review of functional neuroimaging studies. Brain Research Reviews, 45, 96-103.

Barnes, G. R., \& Hillebrand, A. (2003). Statistical flattening of MEG beamformer images. Human Brain Mapping, 18, 1-12.

BisHop, S. J. (2007). Neurocognitive mechanisms of anxiety: An integrative account. Trends in Cognitive Sciences, 11, 307-316.

Critchley, H. D., Daly, E. M., Phillips, M., Brammer, M., BullMORE, E., Williams, S. C., ET AL. (2000). Explicit and implicit neural mechanisms for processing of social information from facial expres- sions: A functional magnetic resonance imaging study. Human Brain Mapping, 9, 93-105.

Davis, M., \& Whalen, P. J. (2001). The amygdala: Vigilance and emotion. Molecular Psychiatry, 6, 13-34.

Eimer, M., \& Holmes, A. (2007). Event-related brain potential correlates of emotional face processing. Neuropsychologia, 45, 15-31.

GLÄSCHER, J., \& AdOLPHS, R. (2003). Processing of the arousal of subliminal and supraliminal emotional stimuli by the human amygdala. Journal of Neuroscience, 23, 10274-10282.

Hadjipapas, A., Adjamian, P., Swettenham, J. B., Holliday, I. E., \& BARNES, G. R. (2007). Stimuli of varying spatial scale induce gamma activity with distinct temporal characteristics in human visual cortex. NeuroImage, 35, 518-530.

HamanN, S., \& CANLI, T. (2004). Individual differences in emotion processing. Current Opinion in Neurobiology, 14, 233-238.

Hariri, A. R., Mattay, V. S., Tessitore, A., Fera, F., \& Weinberger, D. R. (2003). Neocortical modulation of the amygdala response to fearful stimuli. Biological Psychiatry, 53, 494-501.

Hillebrand, A., Singh, K. D., Holliday, I. E., Furlong, P. L., \& BARNES, G. R. (2005). A new approach to neuroimaging with magnetoencephalography. Human Brain Mapping, 25, 199-211.

Huppertz, H. J., Otte, M., Grimm, C., Kristeva-Feige, R., MergNER, T., \& LUKING, C. (1998). Estimation of the accuracy of a surface matching technique for registration of EEG and MRI data. Electroencephalography \& Clinical Neurophysiology, 106, 409-415.

Ioannides, A. A., Poghosyan, V., Dammers, J., \& Streit, M. (2004). Real-time neural activity and connectivity in healthy individuals and schizophrenia patients. NeuroImage, 23, 473-482.

Kawasaki, H., Adolphs, R., Kaufman, O., Damasio, H., Damasio, A. R., Granner, M., ET AL. (2001). Single-neuron responses to emotional visual stimuli recorded in human ventral prefrontal cortex. $\mathrm{Na}$ ture Neuroscience, 4, 15-16.

KNyAZEv, G. (2007). Motivation, emotion, and their inhibitory control mirrored in brain oscillations. Neuroscience \& Biobehavioral Reviews, 31, 377-395.

LeDoux, J. [E.] (1998). Fear and the brain: Where have we been, and where are we going? Biological Psychiatry, 44, 1229-1238.

LeDoux, J. E. (2000). Emotion circuits in the brain. Annual Review of Neuroscience, 23, 155-184.

LEWIS, M. D. (2005). Bridging emotion theory and neurobiology through dynamic systems modeling. Behavioral \& Brain Sciences, 28, 169-245.

LiU, L., \& IoAnnides, A. A. (2006). Spatiotemporal dynamics and connectivity pattern differences between centrally and peripherally presented faces. Neurolmage, 31, 1726-1740.

Luo, Q., Holroyd, T., Jones, M., Hendler, T., \& Blair, J. (2007). Neural dynamics for facial threat processing as revealed by gamma band synchronization using MEG. NeuroImage, 34, 839-847.

Maratos, F. A., Anderson, S. J., Hillebrand, A., Singh, K. D., \& BARNES, G. R. (2007). The spatial distribution and temporal dynamics of brain regions activated during the perception of object and nonobject patterns. NeuroImage, 34, 371-383.

Markowitsch, H. J. (1998). Differential contribution of the right and left amygdala to affective information processing. Behavioural Neurology, 11, 233-244.

Miller, R. (1991). Cortico-hippocampal interplay and the representation of contexts in the brain. New York: Springer.

Moradi, F., Liu, L. C., Cheng, K., Waggoner, R. A., Tanaka, K., \& IOANNIDES, A. A. (2003). Consistent and precise localization of brain activity in human primary visual cortex by MEG and fMRI. NeuroImage, 18, 595-609.

Nichols, T. E., \& Holmes, A. P. (2002). Nonparametric permutation tests for functional neuroimaging: A primer with examples. Human Brain Mapping, 15, 1-25.

OCHSNER, K. N., \& GROSS, J. J. (2005). The cognitive control of emotion. Trends in Cognitive Sciences, 9, 242-249.

Öhman, A., Carlsson, K., Lundqvist, D., \& Ingvar, M. (2007). On the unconscious subcortical origin of human fear. Physiology \& Behavior, 92, 180-185.

Paré, D., Collins, D. R., \& Pelletier, J. G. (2002). Amygdala oscillations and the consolidation of emotional memories. Trends in Cognitive Sciences, 6, 306-314. 
PessoA, L. (2005). To what extent are emotional visual stimuli processed without attention and awareness? Current Opinion in Neurobiology, 15, 188-196.

Pessoa, L., Japee, S., Sturman, D., \& Ungerleider, L. G. (2006). Target visibility and visual awareness modulate amygdala responses to fearful faces. Cerebral Cortex, 16, 366-375.

Pessoa, L., Kastner, S., \& Ungerleider, L. G. (2002). Attentional control of the processing of neutral and emotional stimuli. Cognitive Brain Research, 15, 31-45.

Phan, K. L., Wager, T., Taylor, S. F., \& Liberzon, I. (2002). Functional neuroanatomy of emotion: A meta-analysis of emotion activation studies in PET and fMRI. Neurolmage, 16, 331-348.

Phelps, E. A., \& LeDoux, J. E. (2005). Contributions of the amygdala to emotion processing: From animal models to human behavior. $\mathrm{Neu}$ ron, 48, 175-187.

Phillips, M. L., Drevets, W. C., Rauch, S. L., \& Lane, R. [D.] (2003). Neurobiology of emotion perception I: The neural basis of normal emotion perception. Biological Psychiatry, 54, 504-514.

Phillips, M. L., Medford, N., Young, A. W., Williams, L., Williams, S. C. R., Bullmore, E. T., ET AL. (2001). Time courses of left and right amygdalar responses to fearful facial expressions. Human Brain Mapping, 12, 193-202.

Price, J. L., \& Amaral, D. G. (1981). An autoradiographic study of the projections of the central nucleus of the monkey amygdala. Journal of Neuroscience, 1, 1242-1259.

Robinson, S. E., \& VRBA, J. (1999). Functional neuroimaging by synthetic aperture magnometry (SAM). In T. Yoshimoto, M. Kotani, S. Kuriki, H. Karibe, \& N. Nakasato (Eds.), Recent advances in biomagnetism (pp. 302-305). Sendai, Japan: Tohoku University Press.

Rogers, R. L., Baumann, S. B., Papanicolaou, A. C., Bourbon, T. W., Alagarsamy, S., \& Eisenberg, H. M. (1991). Localization of the P3 sources using magnetoencephalography and magnetic resonance imaging. Electroencephalography \& Clinical Neurophysiology, 79, 308-321.

Singh, K. D., Barnes, G. R., \& Hillebrand, A. (2003). Group imaging of task-related changes in cortical synchronisation using nonparametric permutation testing. NeuroImage, 19, 1589-1601.

Streit, M., Dammers, J., Simsek-Kraues, S., Brinkmeyer, J., WölWER, W., \& IOANNIDES, A. (2003). Time course of regional brain activations during facial emotion recognition in humans. Neuroscience Letters, 342, 101-104.

Suslow, T., Ohrmann, P., Bauer, J., Rauch, A. V., Schwindt, W., Arolt, V., ET AL. (2006). Amygdala activation during masked presentation of emotional faces predicts conscious detection of threat-related faces. Brain \& Cognition, 61, 243-248.

Tesche, C. D. (1997). Non-invasive detection of ongoing neuronal population activity in normal human hippocampus. Brain Research, 749, 53-60.

Tesche, C. D., \& Karhu, J. (2000). Theta oscillations index human hippocampal activation during a working memory task. Proceedings of the National Academy of Sciences, 97, 919-924.

VRBA, J., \& Robinson, S. E. (2001). Signal processing in magnetoencephalography. Methods, 25, 249-271.

Vuilleumier, P. (2005). How brains beware: Neural mechanisms of emotional attention. Trends in Cognitive Sciences, 9, 585-594.

Vuilleumier, P., Armony, J. L., Driver, J., \& Dolan, R. J. (2003). Distinct spatial frequency sensitivities for processing faces and emotional expressions. Nature Neuroscience, 6, 624-631.

Vuilleumier, P., \& Pourtois, G. (2007). Distributed and interactive brain mechanisms during emotion face perception: Evidence from functional neuroimaging. Neuropsychologia, 45, 174-194.

Whalen, P. J., Kagan, J., Cook, R. G., Davis, F. C., Kim, H., Polis, S., ET AL. (2004). Human amygdala responsivity to masked fearful eye whites. Science, 306, 2061.

Whalen, P. J., Shin, L. M., McInerney, S. C., Fischer, H., Wright, C. I., \& RAUCH, S. L. (2001). A functional MRI study of human amygdala responses to facial expressions of fear versus anger. Emotion, 1, 70-83.

Williams, L. M., Das, P., Liddell, B. J., Kemp, A. H., Rennie, C. J., \& Gordon, E. (2006). Mode of functional connectivity in amygdala pathways dissociates level of awareness for signals of fear. Journal of Neuroscience, 26, 9264-9271.

Williams, L. M., Palmer, D., Liddell, B. J., Song, L., \& Gordon, E. (2006). The "when" and "where' of perceiving signals of threat versus non-threat. NeuroImage, 31, 458-467.

Winston, J. S., Vuilleumier, P., \& Dolan, R. J. (2003). Effects of low-spatial frequency components of fearful faces on fusiform cortex activity. Current Biology, 13, 1824-1829.

Woods, R. P. (1996). Modeling for intergroup comparisons of imaging data. NeuroImage, 4, S84-S94.

Wright, C. I., Fischer, H., Whalen, P. J., McInerney, S. C., Shin, L. M., \& RAUCH, S. L. (2001). Differential prefrontal cortex and amygdala habituation to repeatedly presented emotional stimuli. NeuroReport, 12, 379-383.

Yang, T. T., Menon, V., Eliez, S., Blasey, C., White, C. D., Reid, A. J., ET AL. (2002). Amygdalar activation associated with positive and negative facial expressions. NeuroReport, 7, 1737-1741.

ZALD, D. H. (2003). The human amygdala and the emotional evaluation of sensory stimuli. Brain Research Reviews, 41, 88-123.

(Manuscript received February 4, 2008; revision accepted for publication November 14, 2008.) 